\title{
Erste Kristallstruktur eines Selenans; Metall(II)-Komplexe mit dem 2,4,6-Tris(trifluormethyl)selenophenolat-Liganden
}

\author{
Dieter Labahn, Frank Michael Bohnen, Regine Herbst-Irmer, Ehmke Pohl, Dietrnar Stalke und Herbert W. Roesky* \\ Göttingen, Institut für Anorganische Chemie der Universität
}

Bẹi der Redaktion cingegangen am 7. Mai 1993.

Professor Reinhard Schmutzler zum 60. Geburtstage gewidmet

Inhaltsübersicht. $\mathrm{R}_{\mathrm{f}} \mathrm{ScH} \mathbf{I}$ entsteht in einer Eintopfsynthese bei der Reaktion von $R_{\mathrm{f}} \mathrm{L}$ i $\left[\mathrm{R}_{\mathrm{f}}=2,4,6\right.$-Tris(trifluormethyl)phenyl] mit elementarem Selen und anschließender Umsetzung mit $\mathrm{HBF}_{4}$, Mit 1 konnte zum ersten Mal die Kristallstruktur eines Selenans bestimmt werden. Bei der Umsetzung von $1 \mathrm{mit}$
$\mathrm{M}\left[\mathrm{N}\left(\mathrm{SiMe}_{3}\right)_{2}\right]_{2}(\mathrm{M}=\mathrm{Zn}, \mathrm{Pb})$ im Molverhältnis 2:1 erhält man die Mctallselenophenolate $/ \mathrm{n}\left(\mathrm{SeR}_{f}\right)_{2} \cdot \mathrm{HN}\left(\mathrm{SiMc}_{3}\right)_{2} \quad 2$ und $\left[\mathrm{Pb}\left(\mathrm{SeR}_{\mathrm{f}}\right)_{2}\right]_{2}$ 3. 2 kristallisiert als Hexamethyldisilazan-Addukt, während 3 als Dimer vorliegt.

\section{The First Structure of a Selenane; Metal(II) Complexes with the 2,4,6-Tris(trifluoromethyl)- selenophenolato Ligand}

Abstract. $\mathrm{R}_{\mathrm{F}} \mathrm{SeH} 1$ is obtaincd in a single pot reaction of $\mathrm{R}_{\mathrm{l}} \mathrm{Li}$ $\left[\mathbf{R}_{\mathrm{f}}=2,4,6\right.$-tris(trifluoromcthyl)phenyl] with elemental selenium and following treatment with $\mathrm{HBF}_{4}$. 1 gave the first crystal structurc of a sclenane. Reaction of 1 with $\mathrm{M}\left[\mathrm{N}\left(\mathrm{SiMe}_{3}\right)_{2}\right]_{2}$ $(\mathrm{M}=\mathrm{Zn}, \mathrm{Pb})$ in the molar ratio of $2: 1$ yielded the metal sele- nophenolates $\mathrm{Zn}\left(\mathrm{SeR}_{\mathrm{l}}\right)_{2} \cdot \mathrm{HN}\left(\mathrm{SiMe}_{3}\right)_{2} 2$ and $\left[\mathrm{Pb}\left(\mathrm{SeR}_{\mathrm{f}}\right)_{2}\right]_{2}$ 3. 2 crystallizes as hexamethyldisilazane adduct, 3 as a dimer.

Keywords: Selenane; bis(sclenophenolato)metal complexes; crystal structures

\section{Einleitung}

Ionische Metallthiophenolat- und -selenophenolat-Komplexe werden schon seit langem als Modellverbindungen zur Aufklärung der Koordination von aktiven Metallzentren in Metalloproteinen herangezogen [1 - 4]. Steigendes Interesse finden auch die neutralen Metallchalkogenophenolate, nachdem man vor einigen Jahren ihre Bedeutung als Einkomponentenvorstufen in der MOCVD-Technik erkannte [5-8]. Vor allem die Metallverbindungen der 2. Nebengruppe des Periodensystems eignen sich zur Abscheidung dünner Metallsulfid- und -selenidschichten, dic z. B. als II/VI-Halbleiter von Interesse sind. Voraussetzung ist allerdings die Verwendung geeigneter Liganden, die eine Kondensation verhindern, da sonst die Metallchalkogenophenolate nicht ausreichend flüchtig sind [9].

Der 2,4,6-Tris(trifluormethyl)phenylligand [10] hat sich als sehr gecignet erwiesen, reaktive und ungewöhnliche Hauptgruppenelementzentren durch seinen sterischen Anspruch und seine elektronenziehenden Eigenschaften zu stabilisieren [11-14]. Nachdem wir diesen Liganden erfolgreich bei der Synthese niedermolekularer Metall(I)-thiophenolate eingesetzt hatten [15-16], wollten wir nun flüchtige Metallselenophenolate synthetisiercn und diese zur Abscheidung von Metallselenidschichten einsetzen [17].

\section{Beschreibung der Versuche}

Bei der Umsetzung von $R_{r} L i$ [18] mit einem Überschuß roten oder schwarzen Selens bildet sich durch Insertion des Selens in die Kohlenstoff-Lithium-Bindung $\mathrm{R}_{\mathrm{f}} \mathrm{Seli}$. Die Protonierung mit $\mathrm{HBF}_{4}$ ergibt 1 , das durch Destillation in $40 \%$ iger Ausbeute erhalten werden kann.

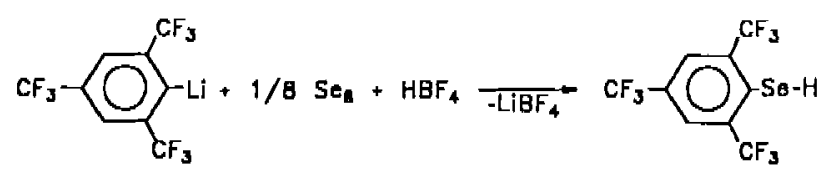


1 ist eine bei Raumtemperatur klare Flüssigkeit mit stechendem Geruch, die bei $19^{\circ} \mathrm{C}$ fest wird. Sie ist hydrolysestabil und nicht mit Wasser mischbar, wird aber durch Oxidationsmittel leicht zum Bis[2,4,6-tris(trifluormethyl)-phenyl]disclenan oxidiert. So verfärbt sich 1 bei Kontakt mit Luftsauerstoff im Verlauf mehrerer Stunden zunehmend orange.

$2 \mathrm{R}_{\mathrm{f}} \mathrm{ScH}+1 / 2 \mathrm{O}_{2} \rightarrow \mathrm{R}_{\mathrm{f}} \mathrm{Sc}-\mathrm{SeR}_{\mathrm{f}}+\mathrm{H}_{2} \mathrm{O}$

Auch unter Lichteinfluß wird 1 in einer Inertgasatmosphäre zum Diselenan oxidiert. Allerdings läuft dann die Oxidation sehr viel langsamer ab. Im ${ }^{1} H$-NMR-Spektrum findet man das Signal für das Se- $\mathrm{H}$-Proton bei $\delta=2,36 \mathrm{ppm}$. Es ist gegenüber den bisher synthetisierten 2,4,6-Tris(trifluormethyl)chalkogenophenolen $\mathrm{R}_{t} \mathrm{OH}$ und $\mathbf{R}_{\mathrm{f}} \mathrm{SH}$ erwartungsgemäß hochfeldverschoben ( $\delta=6,4$ ppm bzw. 4,3 ppm), woraus eine geringere Acidität und Reaktivität gegenüber Nukleophilen abgeleitet werden kann. Durch die Kopplung mit den sechs o-Fluoratomen erscheint das Resonanzsignal bei $\delta=2,36 \mathrm{ppm}$ als Septett mit einer Kopplungskonstanten von ${ }^{5} J_{(\mathrm{H}, \mathrm{F})}=3,2 \mathrm{~Hz}$. Aus den Selen-Satelliten ergibt sich eine Kopplungskonstante des Selens mit dem aciden Proton von ${ }^{1} J_{(H . S e)}=32,5 \mathrm{~Hz}$. In der gleichen Größenordnung koppelt das Selenatom mit den aromatischen Wasserstoffatomen. Aus den Selen-Satelliten des Singuletts bei $\delta=7,63 \mathrm{ppm}$ kann eine Kopplungskonstante von ${ }^{4} \mathrm{~J}_{(\mathrm{H}, \mathrm{Sc})}=27,0 \mathrm{~Hz}$ ermittelt werden.

2 bildet sich bei der Reaktion von Zink(II)-bis(hexamethyldisilylamid) mit zwei Äquivalenten 1. Es handelt sich um einen farblosen, luft- und hydrolyscenpfindlichen Feststoff, der bereits bei $104^{\circ} \mathrm{C}$ schmilzt und in allen gebräuchlichen organischen Lösungsmitteln sehr gut loslich ist.

Die Koordination eines Äquivalents Hexamethyldisilazan je monomerer Einheit wird u.a. durch das ${ }^{1} \mathrm{H}-$ NMR-Spektrum bestätigt, da die Signale bei $\delta=-0,12$ und $3,38 \mathrm{ppm}$ aufgrund ibrer chemischen Verschiebung und ihrer Intensitäten den Methylprotonen und dem $\mathrm{N}-\mathrm{H}$-Proton zugeordnet werden können. Im IR-Spektrum beobachtet man neben den charakteristischen starken Banden des $\mathrm{R}_{\mathrm{f}}$-Liganden die Absorptionsbande der $\mathrm{N}-\mathrm{H}$-Schwingung bei $3171 \mathrm{~cm}^{-1}$. Um die spektroskopischen Ergebnisse zu bestätigen, wurde 2 bei $-30^{\circ} \mathrm{C}$ aus Toluol kristallisiert und eine Röntgenstrukturanalyse durchgeführt.

Die Koordination des Hexamethyldisilazans an das Zinkatom ist recht ungewöhnlich, wenn man bedenkt, daß seine Basizität und damit die Elektronendonoreigenschaften durch die beiden Trimethylsilylgruppen verringert wird. In Übereinstimmung mit dieser Überlegung gibt es in der Literatur noch kein durch eine Röntgenstrukturanalyse belegtes Beispiel für eine Koordination von Hexamethyldisilazan an ein Metallatom. Dennoch ist die Bindung an das Zinkatom so fest, daß 2 zur quantitativen Eliminierung des Hexamethyldisilazans bei $10^{-3} \mathrm{~Pa}$ auf $95^{\circ} \mathrm{C}$ erhitzt werden muß.

Unter diesen Bedingungen wird farbloses 2 a erhalten, das sich bei $142^{\circ} \mathrm{C}$ ohne zu schmelzen zersetzt. Im Gegensatz zu $\mathbf{2}$ ist $\mathbf{2 a}$ in unpolaren Lösungsmitteln wie Toluol und n-Hexan nicht löslich.

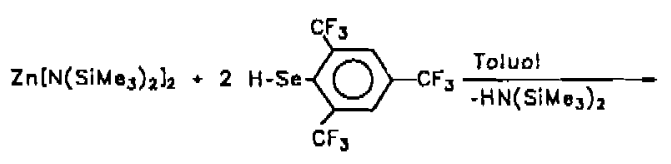

1

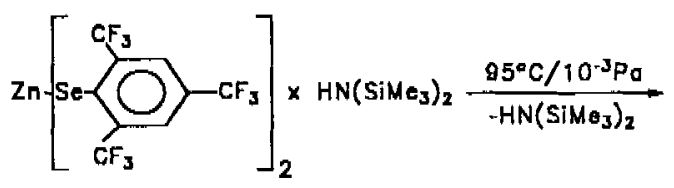

$\underline{2}$

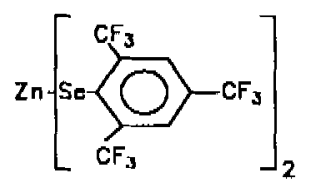

2

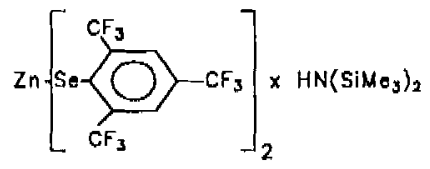

$\underline{2 a}$

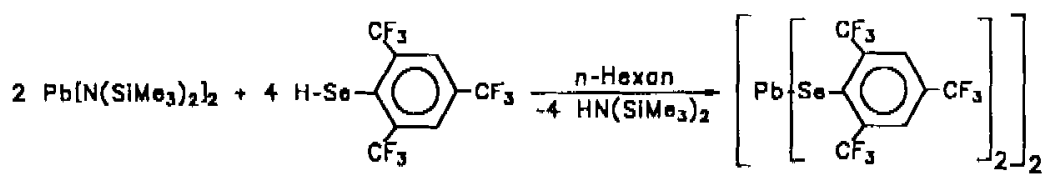


Bei der Reaktion von Blei(II)-bis(hexamethyldisilylamid) mil zwei Äquivalenten $\mathbf{1}$ erhält man gelbes $\mathbf{3}$ in 94\%iger Ausbeute.

3 zersetzt sich langsam unter Luft- und Feuchtigkeitseinwirkung, ist aber unter Inertgasatmosphäre sowohl in Lösung als auch als Feststoff stabil. Aus 'Toluol wurden bei $-30^{\circ} \mathrm{C}$ für eine Röntgenstrukturanalyse geeignete Einkristalle erhalten. 3 ist das erste rontgenstrukturanalytisch untersuchte neutrale Blei(II)-selenophenolat. Daneben ist noch eine ionische Verbindung bekannt [19].

\section{Diskussion der Kristallstrukturen}

$\mathrm{R}_{l} \mathrm{SeH}$ kristallisiert in einer zick-zack-förmigen Fischgrätenanordnung, die vermutlich durch Wasserstoffbrücken stabilisiert wird (Abb. 1). Der intermolekulare Abstand $\mathrm{Sc}-\mathrm{Se}$ ist mit 351,9 pm deutlich kürzer als die Summe der van der Waals Radien von 400 pm [20]. Das an das Selenatom gebundene Wasserstoffatom konnte jedoch nicht lokalisiert werden. Wichtige Bindungslängen und -winkel von 1 sind in Tabelle 1 zusammengefaßt.

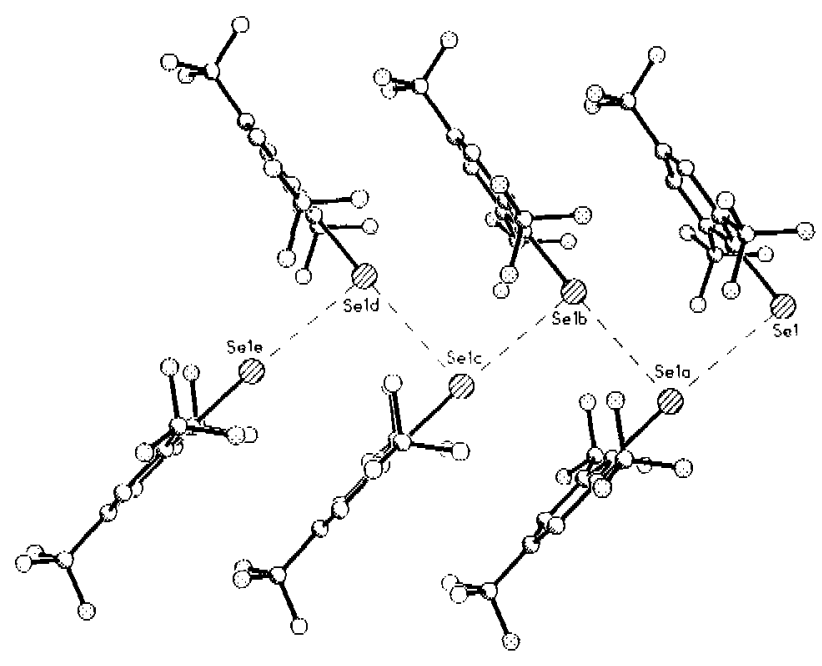

Abb. 1 Kristallstruktur von 1
Tabelle 1 Ausgewähltc Bindungslängen [pm] und -winkel $\left[{ }^{\circ}\right]$ von 1

\begin{tabular}{llll}
\hline$S e(1)-C(1)$ & $191,0(3)$ & $C(1)-C(2)$ & $139,2(4)$ \\
$C(1)-C(6)$ & $139,7(4)$ & $S e(1)-S c(1 a)$ & $351,9(1)$ \\
$C(2)-C(1)-C(6)$ & $117,6(3)$ & $C(2)-C(1)-S e(1)$ & $121,7(2)$ \\
$C(6)-C(1)-\operatorname{Se}(1)$ & $120,7(2)$ & & \\
\hline
\end{tabular}

Symmctrietransformationen für die äquivalenten Atome: $0,5-x, 0,5+y, 0,5-z$

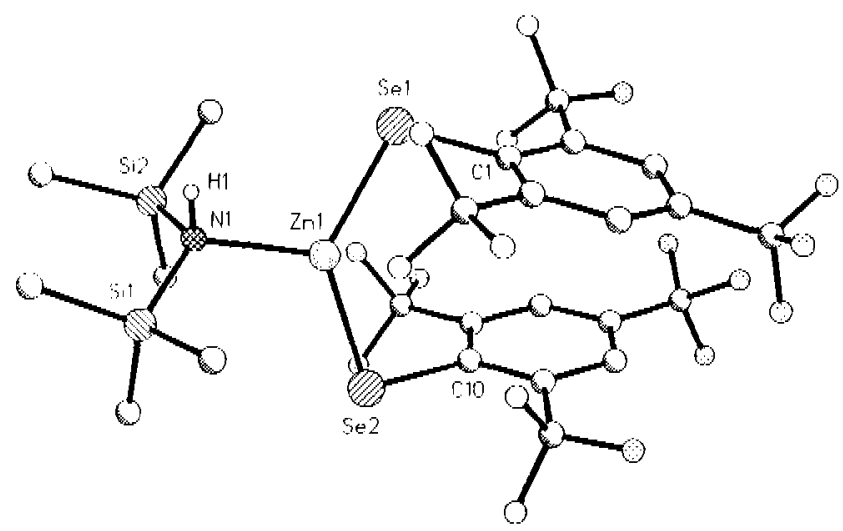

Abb. 2 Kristallstruktur von 2

Die Kristallstruktur von $\mathrm{Zn}\left(\mathrm{SeR}_{f}\right)_{2} \cdot \mathrm{HN}\left(\mathrm{SiMe}_{3}\right)_{2}$ ist in Abb. 2 dargestellt. Ausgewählte Bindungslängen und -winkel sind in Tabelle 2 gegeben. Zink ist annähernd trigonal planar von den beiden Selenatomen und dem Stickstoff des koordinierenden Hexamethyldisilazans umgeben. Die Summe der Bindungswinkel beträgt $359,9^{\circ}$. Die $\mathrm{Zn}$-Se-Abstände betragen 235,2 und $236,7 \mathrm{pm}$ und sind damit etwas kürzer als bisher gefundene $Z_{n}-S e-A b s t a ̈ n-$ de $(243,4-256,8 \mathrm{pm})[3,21-23]$. Die kürzeren Bindungslängen resultieren aus der niedrigeren Koordinationszahl des Zinkatoms. Bemerkenswert an der Kristallstruktur ist das koordinierende Hexamethyldisilzanmolekül. Der Abstand $\mathrm{Zn}-\mathrm{N}$ ist mit 207,4 pm gut vergleichbar mit dem Mittelwert aus der Cambridge Structural Database von $210 \mathrm{pm}$ für $\mathrm{Zn}-\mathrm{N}$-Einfachbindungen

Tabelle 2 Ausgewählte Bindungslängen [prn] und -winkel $\left[{ }^{\circ}\right]$ von 2

\begin{tabular}{llll}
\hline $\operatorname{Zn}(1)-\mathrm{N}(1)$ & $207,4(4)$ & $\mathrm{Zn}(1)-\mathrm{Se}(2)$ & $235,22(8)$ \\
$7 \mathrm{n}(1)-\mathrm{Se}(1)$ & $236,65(8)$ & $\mathrm{Se}(1)-\mathrm{C}(1)$ & $192,7(5)$ \\
$\mathrm{Se}(2)-\mathrm{C}(10)$ & $193,1(5)$ & $\mathrm{N}(1)-\mathrm{Si}(1)$ & $182,0(4)$ \\
$\mathrm{N}(1)-\mathrm{Si}(2)$ & $182,1(4)$ & $\mathrm{N}(1)-\mathrm{H}(1)$ & $89,5(10)$ \\
& & & \\
$\mathrm{N}(1)-\mathrm{Zn}(1)-\mathrm{Se}(2)$ & $116,24(11)$ & $\mathrm{N}(1)-\mathrm{Zn}(1)-\mathrm{Se}(1)$ & $110,22(11)$ \\
$\mathrm{Se}(2)-\mathrm{Zn}(1)-\mathrm{Se}(1)$ & $133,42(3)$ & $\mathrm{C}(1)-\mathrm{Se}(1)-\mathrm{Zn}(1)$ & $102,41(14)$ \\
$\mathrm{C}(10)-\mathrm{Se}(2)-\mathrm{Zn}(1)$ & $99,12(13)$ & $\mathrm{Si}(1)-\mathrm{N}(1)-\mathrm{Si}(2)$ & $120,6(2)$ \\
$\mathrm{Si}(1)-\mathrm{N}(1)-\mathrm{Zn}(1)$ & $113,1(2)$ & $\mathrm{Si}(2)-\mathrm{N}(1)-\mathrm{Zn}(1)$ & $110,9(2)$ \\
$\mathrm{Si}(1)-\mathrm{N}(1)-\mathrm{H}(1)$ & $105,4(30)$ & $\mathrm{Si}(2)-\mathrm{N}(1)-\mathrm{H}(1)$ & $102,6(30)$ \\
$\mathrm{Zn}(1)-\mathrm{N}(1)-\mathrm{H}(1)$ & $101,6(30)$ & & \\
\hline
\end{tabular}


(1525 Einträge) [24]. Das Stickstoffatom besitzt tetracdrische Umgebung, wobei das Wasserstoffatom cindeutig aus der Differenzelektronendichte lokalisiert werden konnte. Die Bindungslängen $\mathrm{Si}-\mathrm{N}$ sind mit durchschnittlich $182,1 \mathrm{pm}$ deutlich länger als der Wert aus Elektronenbeugungsdaten $(173,5 \mathrm{pm})$ für das freie Hexamethyldisilzan [25]. Das kann damit erklärt werden, daß die Elektronendichte am Stickstoffatom durch den EinHuß des Metallatoms verringert wird und so die $\mathrm{Si}-\mathrm{N}$-Bindung geschwächt wird.

In der Kristallstruktur von $\mathrm{Zn}\left(\mathrm{SR}_{\mathrm{f}}\right)_{2} \cdot 2 \mathrm{THF}$ ist das Zink tetracdrisch koordiniert, wohingegen die Anordnung der $R_{f}$ Liganden ähnlich ist [15].

$\left[\mathrm{Pb}\left(\mathrm{SeR}_{\mathrm{t}}\right)_{2}\right]_{2}$ liegt im Kristall als Dimer vor. Ausgewählte Bindungslängen und -winkel sind in Tabelle 3 gegeben. Die zentrale Einheit besteht aus einem viergliedrigen Ring mit alternierender $\mathrm{Pb}-\mathrm{Se}-\mathrm{Anordnung}$ (Abb. 3). Der viergliedrige Ring ist aufgrund eines kristallographischen Inversionszentrums in der Ringmitte exakt planar. Die beiden Bleiatome bilden mit je drei Selenatomen zwei kantenverbrückte trigonale Pyramiden. Die dadurch entstchende Koordinationslücke am Blei wird von einem Toluolmolekül besetzt. Die Abstände $\mathrm{Pb}-\mathrm{Se}$ innerhalb des vierglicdrigen Rings sind mit 287,6 pm ( $\mathrm{Pb} 1-\mathrm{Se} 1 \mathrm{a})$ und $297,0 \mathrm{pm}(\mathrm{Pb} 1$ - Se1) signifikant länger als der terminale $\mathrm{Pb}-\mathrm{Se} 2-\mathrm{Abstand}$ von $271,3 \mathrm{pm}$. Dieser Abstand ist mit

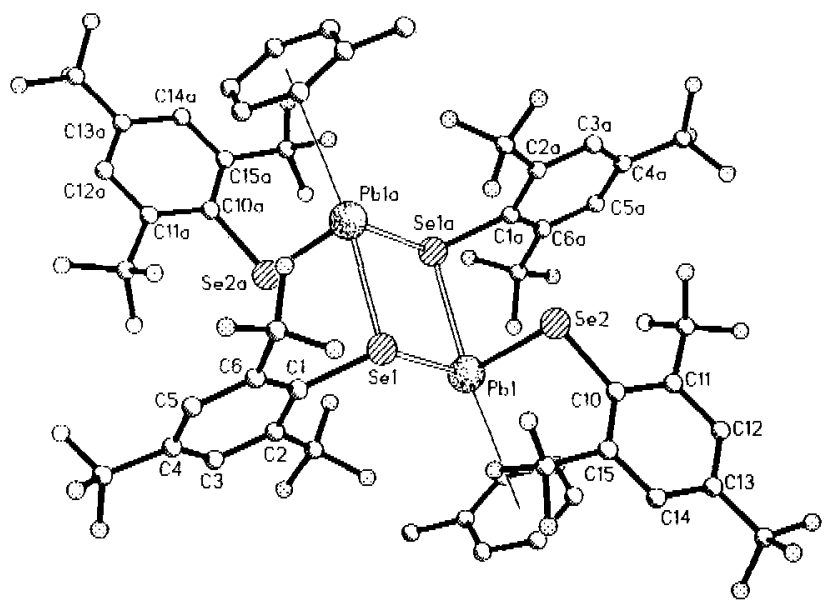

Abb. 3 Kristallstruktur von 3 den Werten für das $\left[\mathrm{Pb}\left(\mathrm{SeC}_{6} \mathrm{H}_{5}\right)_{3}\right]$-Anion vergleichbar $(272,7-276,2 \mathrm{pm})[19]$.

\section{Expcrimenteller Teil}

Die folgenden Meßgeräte wurden zur Datensammlung verwendet:

IR-Spektren, Biorad FTS-7; NMR-Spektren, Bruker AM 250 und Bruker WP 80 SY, Massenspektren, Varian MAT CH5; Schmelzpunkte, Büchi 510 in abgeschmolzenen Glaskapillaren ohne Korrektur. Die Elementaranalysen wurden vom analylischen Laboratorium des Instituts für Anorganische Chemic der Universität Göttingen und vom Mikroanalytischen Laboratorium Bcller, Gôttingen, durchgeführt.

R,SeH 1. Zu eincr Lösung von $56,42 \mathrm{~g}(0,20 \mathrm{~mol}) 2,4,6$ Tris(trifluormethyl)benzol $R_{f} \mathrm{H}$ in $200 \mathrm{ml} \quad \mathrm{Et}_{2} \mathrm{O}$ werden $85 \mathrm{ml}$ $(0,20 \mathrm{~mol})$ einer $\mathrm{n}$-Butyllithium-Lösung $(2,36 \mathrm{M}$ in $\mathrm{n}$-Hexan) langsam zugetropft, damit das Lösungsmittel schwach siedet. Man rühtt $6 \mathrm{~h}$ bei Raumtemperatur und fügt anschließend $18,95 \mathrm{~g}(0,24 \mathrm{~mol})$ Selen im Uberschuß hinzu. Nach weiteren $6 \mathrm{~h}$ wird die Lösung auf $-78^{\circ} \mathrm{C}$ gekühlt und mit $27 \mathrm{ml}(0,20 \mathrm{~mol})$ $\mathrm{HBF}_{4} \cdot \mathrm{Et}_{2} \mathrm{O}$ versetzt. Nach $12 \mathrm{~h}$ Rühren unter Lichtausschlu 3 bei Raumtemperatur werden die unlöslichen Bestandteile abfiltriert, die Lösungsmittel im Vakuum (4 kPa) entfernt und der Rückstand zweimal destilliert. Es resultieren $28,88 \mathrm{~g}(0,08 \mathrm{~mol}$, $40 \%$ ) von 1 als farblose Flüssigkeit. Sdp. $46^{\circ} \mathrm{C} / 0,4 \mathrm{~Pa}$, Schmp. $19^{\circ} \mathrm{C}$.

'H-NMR $\left(250,13 \mathrm{MHz}, \mathrm{C}_{6} \mathrm{D}_{6}\right.$, TMS): $\delta 2,36$ (scpt., $1 \mathrm{H}$, $\left.\mathrm{Sc}-\mathrm{H}, \quad \mathrm{J}_{\mathrm{H}_{-19} \mathrm{~g}}=3,2 \mathrm{~Hz},{ }^{\prime} \mathrm{J}_{14} n_{\mathrm{Sc}}=32,5 \mathrm{~Hz}\right), 7,63(\mathrm{~s}, 2 \mathrm{H}$, $\mathrm{H}_{\text {arom., }}{ }^{4} \mathrm{~J}_{\mathrm{I} \text { i }}$ ?/ $\left.\mathrm{Sc}=27,0 \mathrm{~Hz}\right) \mathrm{ppm}$.

${ }^{19} \mathrm{~F}-\{1 \mathrm{H}\}-\mathrm{NMR}\left(75,39 \mathrm{MHz}, \mathrm{C}_{6} \mathrm{D}_{6}, \mathrm{CFCl}_{3}\right) \delta-62,5$ (td, $6 \mathrm{~F}$,

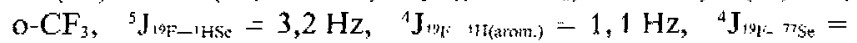
$28,0 \mathrm{~Hz}),-63,6\left(\mathrm{~s}, 3 \mathrm{~F}, \mathrm{p}-\mathrm{CF}_{3}\right)$ ppm.

IR (CsI, rein, cm '): 1626 (st), 1379 (st), 1301 (sst), 1277 (sst), 1268 (sst), 1198 (sst), i 134 (sst), 918 (st), 684 (st).

Massenspcktrum (EI): $\mathrm{m} / \mathrm{z} 362(\mathrm{M}, 28 \%), 342(\mathrm{M}-\mathrm{F}-\mathrm{H}$, 100).

Elementaranalyse für $\mathrm{C}_{9} \mathrm{H}_{3} \mathrm{~F}_{9} \mathrm{Se}(\mathrm{M}=361,06 \mathrm{~g} / \mathrm{mol}): \quad \mathrm{C} 29,3$ (ber. 29,9), H $0,9(0,8)$, Sc $21,4(21,9) \%$.

$\mathbf{Z n}\left(\mathbf{S c R}_{\mathrm{f}}\right)_{2} \cdot \mathbf{H N}\left(\mathrm{SiMe}_{3}\right)_{2} 2.2,02 \mathrm{~g}(5,6 \mathrm{mmol}) \mathbf{1}$ werden zu ciner Lösung von $1,08 \mathrm{~g}(2,8 \mathrm{mmol})$ Zink(II)-bis(hexamethyldisilylamid) in $40 \mathrm{ml}$ Toluol getropft. Man läßt $12 \mathrm{~h}$ unter Lichtausschluß rühren und engt anschließend bis zur Trockene ein. Der Rückstand wird in $60 \mathrm{ml} \mathrm{n}$-Hexan gelöst und die klarc I ösung abgekühlt, bis 2 auskristallisicrt. Nach Abfiltrieren des Feststoffs und Trocknen im Ölpumpenvakuum erhält man $1,23 \mathrm{~g}$ $(1,3 \mathrm{mmol}, 46 \%)$ farbloses 2 . Schmp. $104^{\circ} \mathrm{C}$.

Tabelle 3 Ausgewählte Bindungslängen [pm] und -winkcl $\left[{ }^{\circ}\right]$ von 3

\begin{tabular}{lclc}
\hline $\mathrm{Pb}(1)-\mathrm{Se}(2)$ & $271,27(11)$ & $\mathrm{Pb}(1)-\mathrm{Sc}(1 \mathrm{a})$ & $287,6(2)$ \\
$\mathrm{Pb}(1)-\mathrm{Se}(1)$ & $297,04(13)$ & $\mathrm{Se}(1)-\mathrm{C}(1)$ & $192,5(5)$ \\
& & $\operatorname{Se}(2)-\mathrm{C}(10)$ & $191,5(6)$ \\
& & \\
$\mathrm{Se}(2)-\mathrm{Pb}(1)-\mathrm{Se}(1 \mathrm{a})$ & $87,53(4)$ & $\mathrm{Se}(2)-\mathrm{Pb}(1)-\mathrm{Se}(1)$ & $81,87(3)$ \\
$\mathrm{Se}(1 \mathrm{a})-\mathrm{Pb}(1)-\mathrm{Se}(1)$ & $81,68(4)$ & $\mathrm{C}(1)-\mathrm{Se}(1)-\mathrm{Pb}(1 \mathrm{a})$ & $100,8(2)$ \\
$\mathrm{C}(1)-\mathrm{Se}(1)-\mathrm{Pb}(1)$ & $122,9(2)$ & $\mathrm{Pb}(1 \mathrm{a})-\mathrm{Se}(1)-\mathrm{Pb}(1)$ & $98,32(4)$ \\
$\mathrm{C}(10)-\mathrm{Sc}(2)-\mathrm{Pb}(1)$ & $101,0(2)$ & & \\
\hline
\end{tabular}

Symmetrietransformationen für die äquivalenten Atomc: $a-x,-y+1,-z$ 
Tabelle 4 Kristalldaten, Datensammlung und Verfeinerung von 1, 2 und 3

\begin{tabular}{|c|c|c|c|}
\hline Verbindung & 1 & 2 & 3 \\
\hline Formel & $\mathrm{C}_{9} \mathrm{H}_{3} \mathrm{~F}_{9} \mathrm{Se}$ & $\mathrm{C}_{31} \mathrm{H}_{31} \mathrm{~F}_{18} \mathrm{NSe}_{2} \mathrm{Si}_{2} \mathrm{Zn}$ & $\mathrm{C}_{57} \mathrm{H}_{32} \mathrm{~F}_{36} \mathrm{~Pb}_{2} \mathrm{Sc}_{4}$ \\
\hline Molmasse & 361,07 & 1039,04 & 2131,0 \\
\hline Kristallsystem & Monoklin & Monoklin & Triklin \\
\hline Raumgruppe & $\mathrm{C} 2 / \mathrm{c}$ & $\mathrm{C} 2 / \mathrm{C}$ & $P \overline{1}$ \\
\hline a $(\mathrm{pm})$ & $1714,8(4)$ & $2829,0(3)$ & $1099,2(5)$ \\
\hline$b(\mathrm{pm})$ & $500,7(1)$ & $989,3(2)$ & $1310,6(5)$ \\
\hline$c(p m)$ & $2601,6(6)$ & $2844,0(2)$ & $1383,3(6)$ \\
\hline$\alpha\left(^{\circ}\right)$ & 90 & 90 & $111,75(2)$ \\
\hline$\beta\left({ }^{\circ}\right)$ & $97,04(2)$ & $95,83(1)$ & $101,66(2)$ \\
\hline$\gamma\left({ }^{\circ}\right)$ & 90 & 90 & $109,31(2)$ \\
\hline Zellvolumen $\left(\mathrm{nm}^{3}\right)$ & $2,2169(9)$ & $7,918(2)$ & $1,6226(12)$ \\
\hline $\mathrm{Z}$ & 8 & 8 & 1 \\
\hline$\rho_{\text {ber }}\left(\mathrm{g} / \mathrm{cm}^{3}\right)$ & 2,164 & 1,743 & 2,181 \\
\hline$\mu \mathrm{MoK} \alpha\left(\mathrm{mm}^{-1}\right)$ & 3,493 & 2,629 & 7,566 \\
\hline Kristallgröße $\left(\mathrm{mm}^{3}\right)$ & $0,35 \times 0,3 \times 0,3$ & $0,4 \times 0,4 \times 0,3$ & $0,6 \times 0,6 \times 0,4$ \\
\hline$T_{\text {milax }}, T_{\text {minin }}$ & $0,452,0,389$ & $0,877,0,823$ & $0,729,0,162$ \\
\hline $2 \theta_{\max }\left({ }^{\circ}\right)$ & 55 & 48 & 60 \\
\hline Reflexe gemessen & 4126 & 7030 & 11134 \\
\hline davon unabhängig & 2593 & 6207 & 9262 \\
\hline Anzahl Restraints & 0 & 778 & 282 \\
\hline Anzahl Parameter & 172 & 590 & 488 \\
\hline $\mathrm{R} 1(\mathrm{~F}>4 \sigma(\mathrm{F}))$ & 0,038 & 0,044 & 0,049 \\
\hline wR2 (alle Daten) & 0,107 & 0,091 & 0,142 \\
\hline \multicolumn{4}{|l|}{ Restelektronendichte: } \\
\hline Maximum $\left(10^{6} \mathrm{epm}{ }^{3}\right)$ & 0,43 & 0,60 & 2,69 \\
\hline Minimum $\left(10^{6} \mathrm{cpm}^{-3}\right)$ & $-0,47$ & $-0,42$ & $-3,39$ \\
\hline
\end{tabular}

'H-NMR $\left(250,13 \mathrm{MHz}, \mathrm{C}_{6} \mathrm{D}_{6}, \mathrm{TMS}\right): \delta-0,12\left(\mathrm{~s}, 18 \mathrm{H}, \mathrm{CH}_{3}\right)$, $3,38(\mathrm{~s}, 1 \mathrm{H}, \mathrm{N}-\mathrm{H}), 7,82\left(\mathrm{~s}, 4 \mathrm{H}, \mathrm{H}_{\text {m(m) }}\right)$ ppm.

$\left.{ }^{14} \mathrm{~F}-f^{\prime} \mathrm{H}\right\}-\mathrm{NMR}\left(75,39 \mathrm{MHz}, \mathrm{CD}, \mathrm{CN}, \mathrm{CFCl}_{3}\right) \delta-61,0(\mathrm{~s}, 12 \mathrm{~F}$, $\mathrm{o}-\mathrm{CF}_{3},{ }^{4} \mathrm{~J}_{1 \mathrm{q}}$.

IR (Csl, Nujol, cm ${ }^{1}$ ): $3171(\mathrm{~m}), 1622(\mathrm{st}), 1280$ (sst), 1194 (sst), 1141 (sst), 1092 (st), 917 (st), 845(st), 685 (st).

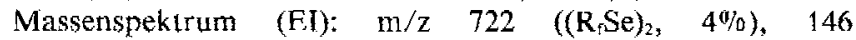
(HN(SiMe $\left.)_{2}-\mathrm{Me}, 100\right)$.

Elementaranalyse für $\mathrm{C}_{24} \mathrm{H}_{23} \mathrm{~F}_{18} \mathrm{NSe}_{2} \mathrm{Si}_{2} \mathrm{Zn}(\mathrm{M}=946,90 \mathrm{~g} / \mathrm{mol})$ : C 29,6 (ber, 30,4), H 2,1 (2,4), N 1,4 (1,5), Zn 7,0 (6,9)\%.

Zn(SeR $)_{2} 2$ a. 2 wird 14 h bei $95^{\circ} \mathrm{C} / 10^{-3} \mathrm{~Pa}$ gehalten, wodurch das Hexamethyldisilazan quantitativ entfernt wird. Es wird Carbloses 2 a erhalten. Zersetzungspunkt $142^{\circ} \mathrm{C}$.

${ }^{\prime} \mathrm{H}-\mathrm{NMR}\left(250,13 \mathrm{MH} \iota, \mathrm{CD}_{3} \mathrm{CN}, \mathrm{TMS}\right): \delta 7,98$ (s) ppm.

$\left.{ }^{19} \mathrm{~F}-f^{\prime} \mathrm{H}\right\}-\mathrm{NMR}\left(75,39 \mathrm{MH} /, \mathrm{CD}_{3} \mathrm{CN}, \mathrm{CFCl}_{3}\right) \delta-59,7(\mathrm{~s}, 12 \mathrm{~F}$, $\left.\mathrm{o}-\mathrm{CF}_{3},{ }^{4} \mathrm{~J}_{19}-{ }_{1 \mathrm{sc}}=20,5 \mathrm{~Hz}\right),-62,3\left(\mathrm{~s}, 6 \mathrm{~F}, \mathrm{p}-\mathrm{CF}_{3}\right) \mathrm{ppm}$.

IR (CsI, Nujol, $\mathrm{cm}$ ): 1623 (st), 1297 (sst), 1282 (sst), 1197 (sst), 1151 (sst), 1134 (sst), 1107 (sst), 1022 (st), 917 (st), 684 (st).

Massenspektrum (EI): $\mathrm{m} / \mathrm{z} 786(\mathrm{M}, 8 \%$ ), 342 (R $\mathrm{Se}-\mathrm{F}, 100)$. Elementaranalyse für $\mathrm{C}_{18} \mathrm{H}_{4} \mathrm{~F}_{18} \mathrm{Se}_{2} \mathrm{Zn} \quad(\mathrm{M}=785,49 \mathrm{~g} / \mathrm{mol})$ : C 27,2 (ber. 27,5$)$, II $0,9(0,5), \operatorname{Zn~} 8,3(8,3) \%$.

$\mathbf{P b}\left(\mathbf{S e R}_{\mathrm{f}}\right)_{2}$ 3, 2,31 g (6,4 mmol) 1 werden bei Raumtemperatur

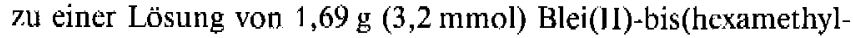
disilylamid) in $50 \mathrm{ml} \mathrm{n}$-Hexan getropft. Man rührt $8 \mathrm{~h}$ unter Lichtausschluß, filtriert den Feststoff ab und wäscht ihn mit $20 \mathrm{ml} \mathrm{n}$-Hexan. Nach dem Entfernen der flüchtigen Bestandteile im Vakuum werden $2,78 \mathrm{~g}(3,9 \mathrm{mmol}, 94 \%) 3$ als gelber Feststoff erhalten. Schmp. $181^{\circ} \mathrm{C}$ (Zersetzung).

${ }^{1} \mathrm{H}-\mathrm{NMR}\left(250,13 \mathrm{MHz}, \mathrm{CD}_{3} \mathrm{CN}, \mathrm{TMS}\right): \delta 8,08(\mathrm{~s}) \mathrm{ppm}$.

${ }^{19} \mathrm{~F}-(1 \mathrm{H}\}-\mathrm{NMR}\left(75,39 \mathrm{MHz}, \mathrm{CD}_{7} \mathrm{CN}, \mathrm{CFCl}_{3}\right) \delta-59,4(\mathrm{~s}, 12 \mathrm{~F}$, $\left.\mathrm{o}-\mathrm{CF}_{3},{ }^{4} \mathrm{~J}_{|\mathrm{q}|}, \mathrm{se}_{\mathrm{se}}=19,3 \mathrm{~Hz}\right),-62,1\left(\mathrm{~s}, 6 \mathrm{~F}, \mathrm{p}-\mathrm{CF}_{3}\right) \mathrm{ppm}$.
IR (CsI, Nujol, $\mathrm{cm}^{-1}$ ): 1621 (st), 1298 (sst), 1279 (sst), t 198 (sst), 1145 (sst), 1110 (sst), 1090 (st), 1023 (st), 917 (st), 684 (st).

Massenspektrum (EI): m/z $928(\mathrm{M}, 6 \%), 323\left(\mathrm{R}_{\mathrm{f}} \mathrm{Se}-2 \mathrm{~F}, 100\right)$. Elcmentaranalyse für $\mathrm{C}_{18} \mathrm{H}_{4} \mathrm{~F}_{18} \mathrm{PbSe}_{2} \quad(\mathrm{M}=927,31 \mathrm{~g} / \mathrm{mol})$ : C 23,1 (ber. 23,3), H 0,7 $(0,4)$, Se $16,7(17,0)$, Pb 8,3 $(8,3) \%$.

Tabelle 5 Atomkoordinaten $\left(\times 10^{4}\right)$ und äquivalente isotrope Auslenkungsparameter $\left(\mathrm{pm}^{2} \times 10^{-1}\right)$ von 1

\begin{tabular}{lrrrr}
\hline Atom & \multicolumn{1}{l}{ x } & \multicolumn{1}{l}{ y } & $\mathrm{U}(\mathrm{cq})$ \\
\hline $\mathrm{Se}(1)$ & $2248(1)$ & $4190(1)$ & $2034(1)$ & $47(1)$ \\
$\mathrm{C}(1)$ & $1912(2)$ & $6780(6)$ & $1514(1)$ & $33(1)$ \\
$\mathrm{C}(3)$ & $2165(2)$ & $9599(7)$ & $801(1)$ & $42(1)$ \\
$\mathrm{C}(5)$ & $881(2)$ & $9444(7)$ & $1055(1)$ & $40(1)$ \\
$\mathrm{C}(2)$ & $2424(2)$ & $7818(7)$ & $1189(1)$ & $38(1)$ \\
$\mathrm{C}(7)$ & $3279(2)$ & $7086(8)$ & $1249(2)$ & $51(1)$ \\
$\mathrm{F}(71)$ & $3669(1)$ & $8380(6)$ & $917(1)$ & $75(1)$ \\
$\mathrm{F}(72)$ & $3638(1)$ & $7621(6)$ & $1720(1)$ & $66(1)$ \\
$\mathrm{F}(73)$ & $3382(1)$ & $4489(5)$ & $1164(1)$ & $64(1)$ \\
$\mathrm{C}(4)$ & $1394(2)$ & $10385(7)$ & $732(1)$ & $42(1)$ \\
$\mathrm{C}(8)$ & $1107(3)$ & $12228(8)$ & $304(1)$ & $59(1)$ \\
$\mathrm{F}(81)$ & $503(2)$ & $13694(7)$ & $405(1)$ & $98(1)$ \\
$\mathrm{F}(82)$ & $870(2)$ & $11009(6)$ & $-130(1)$ & $102(1)$ \\
$\mathrm{F}(83)$ & $1645(2)$ & $13996(6)$ & $210(1)$ & $97(1)$ \\
$\mathrm{C}(6)$ & $1135(2)$ & $7675(6)$ & $1443(1)$ & $35(1)$ \\
$\mathrm{C}(9)$ & $550(2)$ & $6816(8)$ & $1794(1)$ & $48(1)$ \\
$\mathrm{F}(91)$ & $-140(1)$ & $7957(6)$ & $1674(1)$ & $73(1)$ \\
$\mathrm{F}(92)$ & $789(1)$ & $7412(6)$ & $2286(1)$ & $67(1)$ \\
$\mathrm{F}(93)$ & $421(1)$ & $4197(5)$ & $1776(1)$ & $68(1)$ \\
\hline & & & &
\end{tabular}


Tabelle 6 Atomkoordinaten $\left(\times 10^{4}\right)$ und aquivalente isotrope Auslenkungsparameter $\left(\mathrm{pm}^{2} \times 10^{\prime}\right)$ für 2 . U(cq) wird berechnct als cin Drittel der Spur des orthogonalen $U_{\mathrm{ij}}$ Tensors.

\begin{tabular}{|c|c|c|c|c|}
\hline Atom & $x$ & $y$ & $z$ & $U(\mathrm{cq})$ \\
\hline $\mathrm{Zn}(1)$ & $2087(2)$ & $4451(1)$ & $3611(1)$ & $30(1)$ \\
\hline $\operatorname{Se}(1)$ & $1842(2)$ & $4675(1)$ & $4379(1)$ & $51(1)$ \\
\hline$S e(2)$ & $1680(2)$ & $4508(1)$ & $2848(1)$ & $36(1)$ \\
\hline$C(1)$ & $1306(2)$ & $5877(6)$ & $4274(2)$ & $37(3)$ \\
\hline$C(2)$ & $1347(2)$ & $7215(6)$ & $4129(2)$ & $37(3)$ \\
\hline$C(3)$ & $951(2)$ & $8051(6)$ & $4052(2)$ & $39(3)$ \\
\hline$C(4)$ & $508(2)$ & $7569(6)$ & $4125(2)$ & $42(4)$ \\
\hline$C(5)$ & $460(2)$ & $6277(6)$ & $4293(2)$ & $45(3)$ \\
\hline$C(6)$ & $852(2)$ & $5439(6)$ & $4374(2)$ & $40(3)$ \\
\hline$C(7)$ & $1817(2)$ & $7839(5)$ & $4050(2)$ & $47(4)$ \\
\hline$F(71)$ & $2134(1)$ & $7798(4)$ & $4432(1)$ & $63(2)$ \\
\hline $\mathrm{F}(72)$ & $1777(1)$ & $9137(3)$ & $3927(1)$ & $65(2)$ \\
\hline$F(73)$ & $2028(1)$ & $7234(3)$ & $3704(1)$ & $52(2)$ \\
\hline$C(8)$ & $78(2)$ & $8440(6)$ & $4022(2)$ & $59(4)$ \\
\hline$F(81)$ & $-136(2)$ & $8205(8)$ & $3589(2)$ & $117(4)$ \\
\hline$\Gamma(82)$ & $174(2)$ & $9750(4)$ & $4039(3)$ & $85(3)$ \\
\hline$F(83)$ & $-252(2)$ & $8215(7)$ & $4309(3)$ & $105(3)$ \\
\hline$F(84)$ & $33(14)$ & $9089(44)$ & $4429(7)$ & $117(4)$ \\
\hline$F(85)$ & $-334(6)$ & $7840(24)$ & $3903(17)$ & $85(3)$ \\
\hline$F(86)$ & $119(11)$ & $9411(35)$ & $3703(14)$ & $105(3)$ \\
\hline$C(9)$ & $762(2)$ & $4058(6)$ & $4571(2)$ & $52(4)$ \\
\hline$F(91)$ & $310(1)$ & $3875(4)$ & $4650(1)$ & $83(3)$ \\
\hline$F(92)$ & $870(1)$ & $3038(3)$ & $4295(1)$ & $69(3)$ \\
\hline$F(93)$ & $1017(1)$ & $3859(3)$ & $4989(1)$ & $64(3)$ \\
\hline $\mathrm{C}(10)$ & $1038(2)$ & $4311(5)$ & $3013(2)$ & $30(3)$ \\
\hline $\mathrm{C}(11)$ & $720(2)$ & $5400(5)$ & $2973(2)$ & $32(3)$ \\
\hline$C(12)$ & $257(2)$ & $5260(5)$ & $3096(2)$ & $33(3)$ \\
\hline$C(13)$ & $103(2)$ & $4040(6)$ & $3253(2)$ & $36(3)$ \\
\hline$C(14)$ & $400(2)$ & $2936(6)$ & $3275(2)$ & $37(3)$ \\
\hline$C(15)$ & $863(2)$ & $3066(5)$ & $3156(2)$ & $35(3)$ \\
\hline$C(16)$ & $848(2)$ & $6765(5)$ & $2777(2)$ & $41(3)$ \\
\hline$F(161)$ & $962(1)$ & $6672(3)$ & $2334(1)$ & $53(2)$ \\
\hline$F(162)$ & $490(1)$ & $7640(3)$ & $2769(1)$ & $64(2)$ \\
\hline$\Gamma(163)$ & $1215(1)$ & $7356(3)$ & $3030(1)$ & $46(2)$ \\
\hline$C(17)$ & $-386(2)$ & $3882(5)$ & $3396(2)$ & $50(4)$ \\
\hline$\Gamma(171)$ & $-424(1)$ & $2875(5)$ & $3702(2)$ & $76(3)$ \\
\hline$F(172)$ & $-704(1)$ & $3605(7)$ & $3032(1)$ & $87(2)$ \\
\hline$F(173)$ & $-537(2)$ & $4985(5)$ & $3598(2)$ & $94(3)$ \\
\hline$F(174)$ & $-700(9)$ & $4788(33)$ & $3213(14)$ & $76(3)$ \\
\hline$F(175)$ & $-372(11)$ & $4001(50)$ & $3863(3)$ & $87(2)$ \\
\hline$F(176)$ & $-569(12)$ & $2674(21)$ & $3286(16)$ & $94(3)$ \\
\hline$C(18)$ & $1162(2)$ & $1796(5)$ & $3182(2)$ & $49(4)$ \\
\hline$F(181)$ & $1323(1)$ & $1487(3)$ & $2772(1)$ & $62(2)$ \\
\hline $\mathrm{F}(182)$ & $1538(1)$ & $1868(3)$ & $3507(1)$ & $57(2)$ \\
\hline$F(183)$ & $912(1)$ & $722(3)$ & $3301(1)$ & $70(2)$ \\
\hline$N(1)$ & $2805(1)$ & $3997(4)$ & $3652(1)$ & $29(2)$ \\
\hline Si(1) & $3111(1)$ & $4856(2)$ & $3205(1)$ & $30(1)$ \\
\hline $\operatorname{Si}(2)$ & $2908(1)$ & $2208(2)$ & $3775(1)$ & $33(1)$ \\
\hline$C(19)$ & $2988(2)$ & $3901(5)$ & $2646(2)$ & $38(3)$ \\
\hline$C(20)$ & $2873(2)$ & $6602(5)$ & $3144(2)$ & $40(3)$ \\
\hline $\mathrm{C}(21)$ & $3755(2)$ & $4961(6)$ & $3399(2)$ & $46(3)$ \\
\hline$C(22)$ & $2638(2)$ & $1193(5)$ & $3270(2)$ & $44(4)$ \\
\hline $\mathrm{C}(23)$ & $2620(2)$ & $1818(6)$ & $4313(2)$ & $44(3)$ \\
\hline$C(24)$ & $3554(2)$ & $1867(6)$ & $3879(2)$ & $49(3)$ \\
\hline $\mathrm{C}(25)$ & $938(3)$ & $1151(8)$ & 298(4) & $55(3)$ \\
\hline$C(26)$ & $976(3)$ & $-115(9)$ & $93(5)$ & $60(3)$ \\
\hline$C(27)$ & $1417(3)$ & $-636(9)$ & $21(5)$ & $59(3)$ \\
\hline
\end{tabular}

Tabelle 6 (Fortsetzung)

\begin{tabular}{lrrrr}
\hline Atom & $\mathrm{x}$ & $\mathrm{y}$ & $\mathrm{z}$ & $\mathrm{U}(\mathrm{eq})$ \\
\hline $\mathrm{C}(28)$ & $1825(3)$ & $105(8)$ & $162(3)$ & $53(3)$ \\
$\mathrm{C}(29)$ & $1789(2)$ & $1352(9)$ & $377(4)$ & $51(3)$ \\
$\mathrm{C}(30)$ & $1346(3)$ & $1904(11)$ & $428(8)$ & $52(3)$ \\
$\mathrm{C}(31)$ & $1309(4)$ & $3256(9)$ & $641(4)$ & $74(7)$ \\
$\mathrm{C}\left(25^{\prime}\right)$ & $952(4)$ & $1960(12)$ & $368(5)$ & $50(3)$ \\
$\mathrm{C}\left(26^{\prime}\right)$ & $732(4)$ & $776(14)$ & $199(6)$ & $55(4)$ \\
$\mathrm{C}\left(27^{\prime}\right)$ & $1007(6)$ & $-314(14)$ & $77(9)$ & $58(4)$ \\
$\mathrm{C}\left(28^{\prime}\right)$ & $1498(6)$ & $-207(15)$ & $119(10)$ & $58(4)$ \\
$\mathrm{C}\left(29^{\prime}\right)$ & $1716(5)$ & $989(15)$ & $276(10)$ & $52(4)$ \\
$\mathrm{C}\left(30^{\prime}\right)$ & $1444(4)$ & $2041(20)$ & $431(16)$ & $54(4)$ \\
$\mathrm{C}\left(31^{\prime}\right)$ & $1679(6)$ & $3315(14)$ & $597(8)$ & $77(5)$ \\
\hline
\end{tabular}

\section{Röntgenstrukturanalysen von 1, 2 und 3}

Eine Zusammenfassung der Kristalldaten und der Datensammlungen ist in Tabelle 4 gegeben. Atomkoordinaten und äquivalente Auslenkungsparameter für 1,2 und $\mathbf{3}$ sind in dert Tabellen 5, 6 und 7 enthalten. Die Datensammlungen erfolgten mit einem Stoe-Siemens AED Vierk reis-Diffraktometer mit graphitmonochromatisierter Mo-Ka-Strahlung $(\lambda=71,073 \mathrm{pm})$ nach einer ,Learnt Profile Methode [26]. Die Temperatur während der Datensammlung betrug bei $1-85^{\circ} \mathrm{C}$, bei 2 und $3-120^{\circ} \mathrm{C}$. Verbindung 1 wurde wegen des niedrigen Schmelzpunktes bei einer Temperatur von ca. $-30^{\circ} \mathrm{C}$ montiert [27].

Bci allen Verbindungen wurden semiempirische Absorptionskorrekturen durchgeführt. 1 und 2 wurden mit dirckten Mcthoden, 3 mit Patterson- und Fouricrmethoden gelöst [28]. Alle Nicht-Wasserstoffatome wurden anisotrop nach $\mathrm{F}^{2}$ mit dem Kleinste-Quadrate Verfahren verfeincrt [29]. Dic Wasscrstoffatome an den Kohlenstoffatomen wurden geometrisch ideal positioniert und nach einem Reitermodell verfeinert. Das Wasserstoffatom am Selenatom in 1 konnte nicht lokalisiert werden, daher wurde es nicht mit in die Verfeinerung einbezogen. Bei 2 wurde die Position des $\mathrm{N}-\mathrm{H}$-Wasserstoffatoms mit einem $\mathrm{Ab}$ standsrestraint frei verfeinert. Bci dicser Struktur sind die para$\mathrm{CF}_{3}$-Gruppen rotationsfehlgeordnet, was durch das Einfügen einer zweiten Komponente mil einem Besetzungsverhältnis von $0,9: 0,1$ berücksichtigt wurde. Abstandsrestraints wurden für die $1-2$ und $1-3$ Abstände verwendet. Die gegenübcrliegenden Fluoratome wurden mit identischen $\mathrm{U}_{\mathrm{ij}}$-Werten verfeinert. 2 und 3 kristallisieren mit 1 ösungsmittelmolekülen, dic fehlgeordnet sind. In beiden Verfeinerungen wurden Abstandsrestraints zur Auflösung der Fehlordnungen eingcsctzt. Bei 2 wurden zwci Positionen für das lehlgeordnete Toluol auf cine Besetzung von 0,65 bzw. 0,35 verfeineri. Für 3 wurden zwei Positionen für die fehlgeordnete Methylgruppe des koordinierenden Toluolmoleküls mit einer Besetzung von 0,5 verfeinert. Ein zweites Toluolmolekül licgt auf cinem kristallographischen Inversionszentrum. Es wurde als ganzes Molckül mit einer Besetzung von 0,5 verfeinert.

Weiterc Einzelheiten zu den Kristallstrukturuntersuchungen können beim Fachinformationszentrum Karlsruhe, Gcscllschaft für wissenschaftlich-technische Information $\mathrm{mbH}$, D-76344 Eggenstein-Lcopoldshafen, unter Angabe der Hinterlegungsnummer CSD-57278, der Autorennamen und des 7eitschriftenzitats angefordert werden. 
Tabelle 7 Atomkoordinaten $\left(\times 10^{4}\right)$ und äquivalente isotrope Auslenkungsparameter $\left(\mathrm{pm}^{2} \times 10^{\prime}\right)$ für 3. U(eq) wird berechnet als ein Drittel der Spur des orthogonalen $\mathrm{U}_{\mathrm{ij}}$-Tensors.

\begin{tabular}{|c|c|c|c|c|}
\hline Alom & $x$ & $y$ & 2 & $\mathrm{U}(\mathrm{eq})$ \\
\hline $\mathrm{Pb}(1)$ & $756(1)$ & $4134(1)$ & $926(1)$ & $29(1)$ \\
\hline Se(1) & $1594(1)$ & $6547(1)$ & $953(1)$ & $27(1)$ \\
\hline $\mathrm{Se}(2)$ & $2140(1)$ & $3799(1)$ & $-491(1)$ & $33(1)$ \\
\hline$C(1)$ & $1581(6)$ & $7939(5)$ & $2092(4)$ & $25(1)$ \\
\hline$C(2)$ & $1171(6)$ & $7921(5)$ & $2991(5)$ & $29(1)$ \\
\hline $\mathrm{C}(3)$ & $1187(7)$ & $8954(5)$ & $3794(5)$ & $32(1)$ \\
\hline$C(4)$ & $1615(6)$ & $10033(5)$ & $3730(5)$ & $30(1)$ \\
\hline$C(5)$ & $2068(6)$ & $10097(5)$ & $2887(5)$ & $30(1)$ \\
\hline$C(6)$ & $2050(6)$ & $9066(5)$ & $2082(5)$ & $28(1)$ \\
\hline$C(7)$ & $716(8)$ & $6791(6)$ & $3150(6)$ & $37(1)$ \\
\hline $\mathrm{F}(71)$ & $429(7)$ & $6998(4)$ & $4078(4)$ & $64(2)$ \\
\hline$F(72)$ & $1722(5)$ & $6418(4)$ & $3262(4)$ & $43(1)$ \\
\hline$F(73)$ & $-399(4)$ & $5823(4)$ & $2303(4)$ & $42(1)$ \\
\hline$C(8)$ & $1581(8)$ & $11138(6)$ & $4564(6)$ & $40(1)$ \\
\hline$F(81)$ & $2595(6)$ & $12187(4)$ & $4780(4)$ & $58(1)$ \\
\hline$F(82)$ & $386(6)$ & $11193(4)$ & $4194(4)$ & $53(1)$ \\
\hline$F(83)$ & $1670(6)$ & $11128(4)$ & $5536(3)$ & $50(1)$ \\
\hline $\mathrm{C}(9)$ & $2554(7)$ & $9206(5)$ & $1171(6)$ & $35(1)$ \\
\hline $\mathrm{H}(91)$ & $3600(5)$ & $8935(4)$ & $1111(4)$ & $46(1)$ \\
\hline$F(92)$ & $2997(6)$ & $10370(4)$ & $1345(4)$ & $53(1)$ \\
\hline$F(93)$ & $1536(5)$ & $8527(4)$ & $157(3)$ & $42(1)$ \\
\hline$C(10)$ & $3504(6)$ & $3465(5)$ & $263(5)$ & $29(1)$ \\
\hline$C(11)$ & $3415(6)$ & $2276(6)$ & $-209(6)$ & $33(1)$ \\
\hline$C(12)$ & $4453(7)$ & $2023(6)$ & $281(6)$ & $37(1)$ \\
\hline$C(13)$ & $5561(7)$ & $2924(7)$ & $1217(6)$ & $39(1)$ \\
\hline$C(14)$ & $5700(7)$ & $4120(7)$ & $1703(6)$ & $38(1)$ \\
\hline$C(15)$ & $4668(6)$ & $4380(6)$ & $1231(5)$ & $31(1)$ \\
\hline$C(16)$ & $2211(8)$ & $1216(6)$ & $-1242(7)$ & $47(2)$ \\
\hline$F(161)$ & $1009(5)$ & $962(5)$ & $-1074(5)$ & $64(1)$ \\
\hline$F(162)$ & $2370(7)$ & $184(5)$ & $-1524(6)$ & $76(2)$ \\
\hline$F(163)$ & $2055(6)$ & $1397(5)$ & $-2131(4)$ & $60(1)$ \\
\hline$C(17)$ & $6661(10)$ & $2672(10)$ & $1761(8)$ & $57(2)$ \\
\hline$F(171)$ & $6759(11)$ & $2870(11)$ & $2766(7)$ & $123(4)$ \\
\hline$F(172)$ & $7875(6)$ & $3258(7)$ & $1743(8)$ & $91(2)$ \\
\hline$\Gamma(173)$ & $6380(7)$ & $1469(6)$ & $1221(6)$ & $77(2)$ \\
\hline$C(18)$ & $4871(7)$ & $5684(6)$ & $1822(6)$ & $37(1)$ \\
\hline$F(181)$ & $6070(5)$ & $6402(4)$ & $2700(4)$ & $58(1)$ \\
\hline$F(182)$ & $3870(4)$ & $5756(4)$ & $2241(3)$ & $41(1)$ \\
\hline$F(183)$ & $4867(5)$ & $6221(4)$ & $1172(4)$ & $46(1)$ \\
\hline$C(1 S)$ & $1093(10)$ & $3734(9)$ & $3577(9)$ & $70(2)$ \\
\hline$C(2 S)$ & $2529(11)$ & $4438(9)$ & $3949(8)$ & $67(2)$ \\
\hline$C(3 S)$ & $3233(10)$ & $3999(8)$ & $3336(8)$ & $62(2)$ \\
\hline$C(4 S)$ & $2595(9)$ & $2889(8)$ & $2386(8)$ & $57(2)$ \\
\hline$C(5 S)$ & $1196(10)$ & $2187(8)$ & $2016(9)$ & $65(2)$ \\
\hline$C(6 S)$ & $459(10)$ & $2632(9)$ & $2636(9)$ & $70(2)$ \\
\hline$C(7 S)$ & $422(22)$ & $4271(21)$ & $4318(17)$ & $80(4)$ \\
\hline$C\left(7 S^{\prime}\right)$ & $3189(25)$ & $5592(21)$ & $4972(17)$ & $103(8)$ \\
\hline $\mathrm{C}(8 \mathrm{~S})$ & $4741(26)$ & $9861(31)$ & $5303(23)$ & $127(9)$ \\
\hline$C(9 S)$ & $5128(32)$ & $11024(29)$ & $5416(27)$ & $151(13)$ \\
\hline$C(10 S)$ & $5673(30)$ & $11318(23)$ & $4694(29)$ & $145(12)$ \\
\hline$C(11 S)$ & $5962(48)$ & $10509(29)$ & $3925(35)$ & $137(12)$ \\
\hline$C(12 S)$ & $5447(28)$ & $9305(25)$ & $3734(24)$ & $111(8)$ \\
\hline$C(13 S)$ & $4948(41)$ & $9020(28)$ & $4483(31)$ & $117(9)$ \\
\hline$C(14 S)$ & $4342(47)$ & $9601(49)$ & $6169(34)$ & $177(18)$ \\
\hline
\end{tabular}

Wir danken der Deutschen Forschungsgemeinschaft und dem Fonds der Chemischen Industrie für finanzielle Unterstützung.

\section{Literatur}

[1] I. G. Dance, Polyhedron 5 (1986) 1037

[2] P. J. Blower, I. R. Dilworth, Coord. Chem. Rev. 76 (1987) 121

[3] N. Ueyama, T. Sugawara, K. Sasaki, A. Nakamura, S. Yamashita, Y. Wakatsuki, II. Yamazaki, N. Yasuoka, Inorg. Chem. 27 (1988) 741

[4] B. Krebs, G. Henkel, in H.W. Roesky (Hrsg.), Rings, Clusters and Polymers of Main Group and Transition Elements, Elsevier, Amsterdam 1989, S. 439

[5] M. Bochmann, K. Webb, M. Harmann, M. B. Hursthouse, Angew. Chem. 102 (1990) 703; Angew. Chem. Int. Ed. EIIgl. 29 (1990) 638

[6] P. P. Power, S. C. Shoner, Angew. Chem. 102 (1990) 1484; Angew. Chem. Int. Ed. Engl. 29 (1990) 1403

[7] M. Bochmann, A. P. Coleman, K.J. Webb, M. B. Hursthouse, M. Mazid, Angew. Chem. 103 (1991) 975; Angew. Chem. Int. Ed. Engl. 30 (1991) 973

[8] M. Bochmann, K. J. Webb, M. B. Hursthouse, M. Mazid, J. Chem. Soc., Dalton Trans. 1991, 2317

[9] D. Craig, I. G. Dance, R. Garbutt, Angew. Chem, 98 (1986) 178; Angew. Chem. Int. Ed. Engl. 25 (1986) 165

[10] G. E. Carr, R. D. Chambers, T. F. Holmes, D. G. Parker, J. Organomet. Chem. 325 (1987) 13

[11] M. Scholz, II. W. Roesky, D. Stalke, K. Keller, F. T. Edelmann, J. Organomet. Chem. 366 (1989) 73

[12] H.W. Roesky, M. Scholz, M. Nollemeyer, F. T. Edelmann, Inorg. Chem. 28 (1989) 3829

[13] M. Scholz, M. Noltemeyer, H. W. Roesky, Angew. Chem. 101 (1989) 1419; Angew. Chem. Int. Ed. Engl. 28 (1989) 1383

[14] D. Labahn, E. Pohl, R. Herbst-Irmer, D. Stalke, H. W. Roe sky, G. M. Sheldrick, Chem. Ber. 124 (1991) 1127

[15] D. Labahn, S. Brooker, G. M. Sheldrick, H. W. Roesky, Z. anorg. allg. Chem. 610 (1992) 163

[16] D. Labahn, Dissertation, Universität Göttingen 1992

[17] W. Rockensüß, D. Labahn, H. W. Roesky, in Vorbereitung

[18] D. Stalke, K. H. Whitmire, J. Chem. Soc., Chem. Commun. 1990, 833

[19] P. A. W. Dean, J. J. Vitlal, N. C. Payne, Inorg. Chem. 23 (1984) 4232

[20] L. Pauling, The Nature of the Chemical Bond, 3rd cd., Cornell University Press, Ithaca, NY, 1960)

[21] M. A. Ansari, C. H. Mahler, G.S. Chorghade, Y.J. Lu, J. A. Ibers, Inorg. Chem. 29 (1990) 3832

[22] S. Dev, E. Ramli, T. B. Rauchfuss, C. L. Stern, J. Am. Chem. Soc. $112(1990) 6385$

[23] G. Matsubayashi, K. Akiba, T. Tanaka, J. Chem. Soc., Dalton Trans. 1990, 115

[24] F. H. Allen, S. Bellard, M. D. Brice, B. A. Cartwright, A. Doubleday, H. Higgs, T. Hummelink, B. G. Hummelink-Peters, O. Kennard, W. D. S. Motherwell, J. R. Rodgers, D. G, Watson, Acta Crystallogr. B35 (1979) 2331

[25] A. G. Robiette, G. M. Sheldrick, W. S. Sheldrick, B. Beagley, D. W.J. Cruickshank, J. J. Monaghan, B. J. Aylett, I. A. Ellis, Chem. Commun, 1968, 909

[26] W. Clegg, Acta Crystallogr. A37 (1981) 22

[27] T. Kottke, D. Stalke, J. Appl, Crystallogr. 26 (1993) 615

[28] G. M. Sheldrick, Acta Crystallogr. A46 (1990) 467

[29] G. M. Sheldrick, SHELXL-92, Programm zur Kristallstrukturverfeinerung, Universität Göttingen 1992

Anschr. d. Verf:

Prof. Dr, H. W. Roesky

Institut für Anorganische Chemie

Tammannstr. 4

D-37077 Götingen 